\title{
An improved method for the rescue of recombinant Newcastle disease virus
}

\author{
Pheik-Sheen Cheow', Tiong Kit Tan'2, Adelene Ai-Lian Song', Khatijah Yusoff',3 \& Suet Lin Chia ${ }^{1,4, *}$
}

\section{ABSTRACT}

Reverse genetics has been used to generate recombinant Newcastle disease virus with enhanced immunogenic properties for vaccine development. The system, which involves co-transfecting the viral antigenomic plasmid with three helper plasmids into a T7 RNA polymeraseexpressing cell to produce viral progenies, poses a great challenge. We have modified the standard transfection method to improve the transfection efficiency of the plasmids, resulting in a higher titer of virus progeny production. Two transfection reagents (i.e., Lipofectamine and polyethylenimine) were used to compare the transfection efficiency of the four plasmids. The virus progenies produced were quantitated with flow cytometry analysis of the infectious virus unit. The modified transfection method increased the titer of virus progenies compared with that of the standard transfection method.

\section{METHOD SUMMARY}

The standard transfection method was modified by changing the time to perform the co-transfection to produce viral progenies from the transfected mammalian cell line, which improved the titer of virus progenies. The method of viral titer quantification by infecting the cancer cell line was simple and time saving.

\section{KEYWORDS}

Newcastle disease virus $\cdot$ polyethylenimine $\cdot$ reverse genetics $\cdot$ transfection - virus titration

'Department of Microbiology, Faculty of Biotechnology \& Biomolecular Sciences, Universiti Putra Malaysia, 43400 UPM Serdang, Selangor, Malaysia; ${ }^{2}$ MRC Human Immunology Unit, Weatherall Institute of Molecular Medicine, University of Oxford, OX3 9DS, Oxford, UK; ${ }^{3}$ Malaysia Genome Institute, Jalan Bangi, 43000 Kajang, Selangor, Malaysia; ${ }^{4}$ nstitute of Bioscience, Universiti Putra Malaysia, 43400 UPM Serdang, Selangor, Malaysia; *Author for correspondence: suetlin@upm.edu.my

BioTechniques 68: 96-100 (February 2020) 10.2144/btn-2019-0110
Newcastle disease virus (NDV) is a singlestranded, negative-sense, nonsegmented RNA virus [1]. A recombinant NDV (rNDV) can be generated from a cloned cDNA using reverse genetics. The cDNA of the full-length antigenome of the virus, with plasmids encoding for ribonucleoprotein (RNP), are co-transfected into T7 RNA polymerase-expressing cells [2]. The transcription and translation of the RNP in the cells will result in the production of viral progenies, which can then be propagated in embryonated eggs.

The success of the rNDV rescue depends on the efficiency of the transfections of four plasmids (one for fulllength cDNA and three for RNP produced collectively by individual plasmids encoding the $N P, P$ and $L$ genes) into a cell. Several attempts have been carried out to either generate cells that constitutively express the RNP or to reduce the number of plasmids to be transfected $[3,4]$. Both efforts are complicated and time-consuming.

Lipofectamine, a cationic lipid, is commonly used for the transfection of the plasmids into cells. Other transfection reagents, such as cationic polymers (polyethylenimine [PEI]) and inorganic compounds, have also been used for this purpose. However, to the best of our knowledge, no transfection reagents other than Lipofectamine have been used to rescue rNDV. Therefore, it is unclear whether this has not been done or because it was not successful, failed to be reported. Nevertheless, PEI has been used to transfect plasmids for the rescue of influenza virus, a segmented negativesense RNA virus [5]. Here, our aim was to compare the transfection efficiency of Lipofectamine ${ }^{T M} 3000$ (Invitrogen, MA, USA) and PEI with optimized transfection protocol for the rescue of rNDV.

\section{MATERIALS \& METHODS}

Plasmids
pCITE(GFP), a plasmid that is driven by the T7 promoter, was used to monitor T7 polymerase activity in the cells. Plasmid pOLTV5(rAF-GFP), which encodes for the complete antigenome of NDV strain AF2240-I [6], with a green fluorescent protein (GFP), was used to rescue the recombinant NDV (rNDV). Plasmids pCl-neo(NP), pCl-neo(P) and pCl-neo(L), respectively, harboring the $N P, P$ and $L$ genes serve as helper plasmids for the rescue of the virus.

\section{Cell culture}

A Baby Hamster Kidney (BHK)-derived mammalian cell line stably expressing T7 RNA polymerase, known as BSR T7/5 [7], was used in this study. The cell was maintained in Glasgow's Minimum Essential Medium (Sigma-Aldrich, NY, USA) supplemented with $10 \%$ newborn calf serum (Himedia, India), 1\% tryptose phosphate and 1\% Minimum Essential Medium (MEM) nonessential amino acids. Colorectal cancer cell lines, HT29 cells were maintained in Dulbecco's Modified Eagle Medium (Sigma-Aldrich) supplemented with $10 \%$ fetal bovine serum (Sigma-Aldrich). All cell lines were cultured at $37^{\circ} \mathrm{C}$ in a humidified atmosphere with $5 \% \mathrm{CO}_{2}$.

\section{Optimization of PEI}

Prior to transfection, the cytotoxicity of 25-kDa linear PEI (PolySciences, PA, USA) was determined by MTT assay [8]. BSR T7/5 cells $\left(1 \times 10^{5}\right)$ were seeded in 24-well plates and subjected to $\mathrm{PEI}$ treatment at various concentrations. The cell viabilities were measured after $48 \mathrm{~h}$ of PEI treatment. The highest PEI concentration, with at least $80 \%$ relative cell viability, was chosen for subsequent transfections. The PEI/DNA ratio (w/w) optimization was performed by adding various $\mathrm{PEI} / \mathrm{DNA}$ ratio complex into the cells and fluorescence was determined by flow cytometry $48 \mathrm{~h}$ post transfection. 


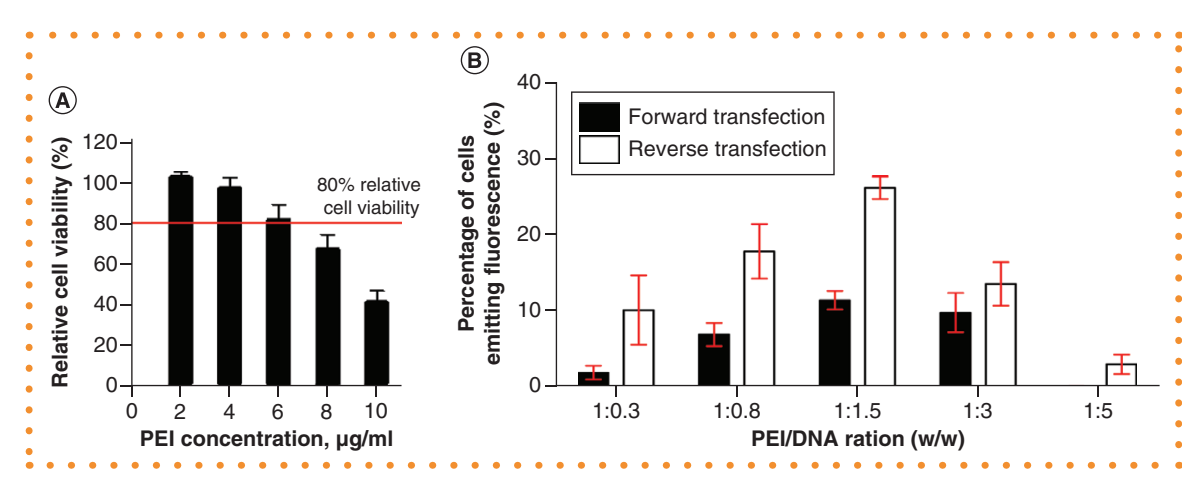

Figure 1. Optimization of BSR T7/5 cells for two parameters, cytotoxicity of polyethylenimine and polyethylenimine/DNA ratio. (A) Relative cell viability of BSR T7/5 after treatment with various PEI concentrations $(\mu \mathrm{g} / \mathrm{ml})$. The cells were treated with PEl at $24 \mathrm{~h}$ post seeding and the relative cell viability was determined after $48 \mathrm{~h}$ of treatment. Cell viability of BSR T7/5 decreased gradually when treated with increasing PEI concentration. The maximum PEI concentration that showed $80 \%$ cell viability was $6 \mu \mathrm{g} / \mathrm{ml}$. (B) Percentage of BSR T7/5 cells emitting fluorescence when transfected with $\mathrm{pCITE}(\mathrm{eGFP}$ ) in either forward or reverse and different PEI/DNA ratios were implemented. The plasmid was transfected at $24 \mathrm{~h}$ post seeding and different PEI mass were added to keep the PEI/ DNA ratio at 1:0.3, 1:0.8, 1:1.5, 1:3 and 1:5. The highest fluorescent percentage was observed for both forward and reverse transfection by using 1:1.5 (PEI/DNA) ratio.

PEI: Polyethylenimine.

Moreover, either forward or reverse transfection was tested to determine the best transfection procedure. Forward transfection was performed by adding the PEI/ DNA complex after $24 \mathrm{~h}$ post seeding, whereas reverse transfection was performed by adding PEI/DNA complex immediately after the cells were seeded.

\section{Evaluation of transfection efficiency}

Cells were trypsinized $48 \mathrm{~h}$ post transfection. Detached cells were centrifuged at $4000 \times g$ for $5 \mathrm{~min}$ at $4^{\circ} \mathrm{C}$ and the resulting cell pellet was resuspended in MACS buffer ( $1 \times$ PBS with $5 \%$ fetal bovine serum and $2 \mathrm{mM}$ EDTA). Cells were then analyzed for fluorescence expression using flow cytometry (Novoexpress, ACEA Biosciences, CA, USA).

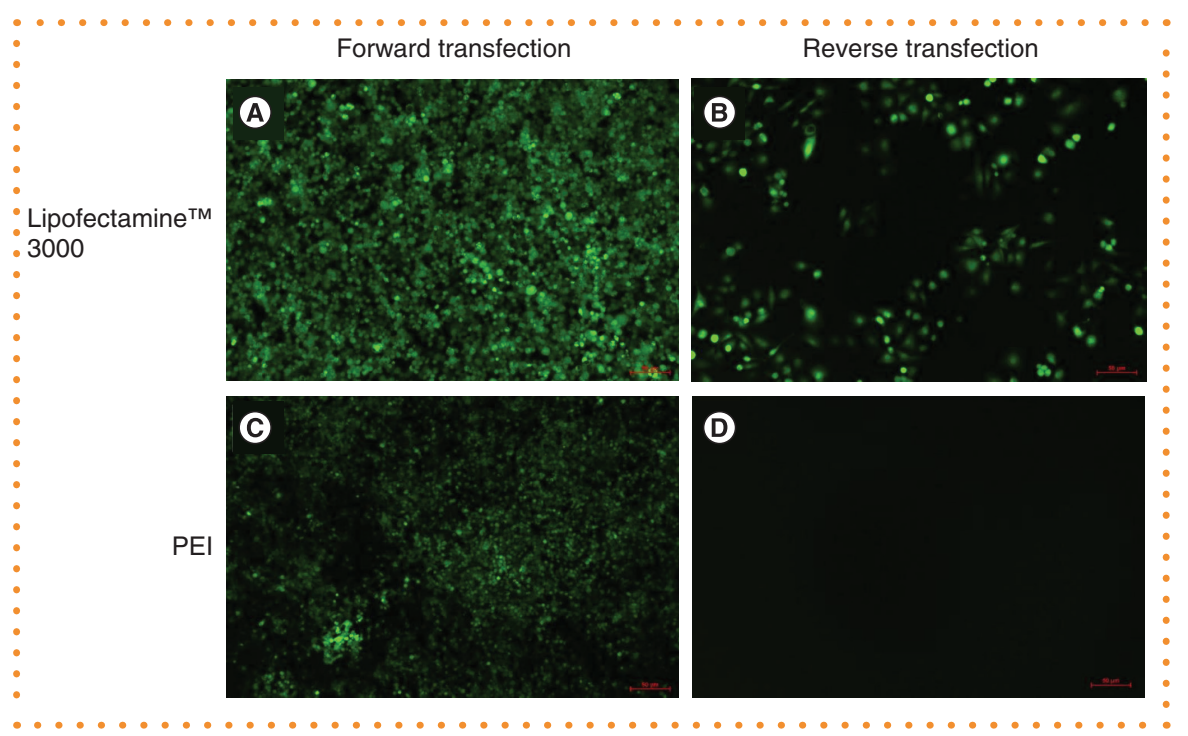

Figure 2. Viral rescue using different types of transfections and transfection reagents in a 24-well plate. (A) Forward transfection, Lipofectamine ${ }^{\mathrm{TM}} 3000$. (B) Reverse transfection, Lipofectamine 3000. (C) Forward transfection, PEI. (D) Reverse transfection, PEI. The fluorescence emitted by the virus were observed using a fluorescence microscope and with $100 \times$ magnification. Rescue of recombinant Newcastle disease virus was repeated in three independent biological replicates (each with three independent technical replicates).

PEl: Polyethylenimine.

\section{Rescue of recombinant NDV}

Two transfection reagents (TRs), PEl and Lipofectamine 3000 , were studied to compare the rescue efficiency of rNDV from cDNA. In the rescue system using Lipofectamine 3000 , the plasmid cocktail (full-length NDV antigenome and the three helper plasmids) was mixed with the TR to form TR/DNA complex according to the instructions of the manufacturer. In the rescue system using $\mathrm{PEI}$, the previously mentioned optimized protocol and TR: DNA ratio ( $\mathrm{w} / \mathrm{w}$ ) was used. The mixture was incubated for $15 \mathrm{~min}$ at room temperature and then it was added to the BSR T7/5 cells. The culture medium containing the virus was harvested 4 days post transfection.

\section{Quantification of viral titer}

HT29 cells were seeded $\left(1 \times 10^{5}\right.$ cells/well) and incubated for $24 \mathrm{~h}$, followed by infection of the diluted virus. The plate was incubated at $37^{\circ} \mathrm{C}$ for $1 \mathrm{~h}$, with 15 -min interval rocking. Then, the infectious media was discarded and complete media was added to the cells. Cells were harvested and paraformaldehydefixed to determine the percentage of fluorescent cells at $12 \mathrm{~h}$ post infection. Based on the fluorescence percentile, the virus titer was calculated as infectious unit/ $\mathrm{ml}(\mathrm{IFU} / \mathrm{ml})$ as shown in the following equation:
$\operatorname{Titer}(\mathrm{IFU} / \mathrm{ml})=$
Number of cells plated $\times(\%$ of GFP postive cells $) \times$ Dilution facto Volume of infectious media $(\mathrm{ml})$

\section{RESULTS \& DISCUSSION}

Several transfection parameters were optimized to obtain the optimum condition for co-transfection. Cytotoxicity of PEl is a prime concern for PEl-mediated gene transfer $[9,10]$. It was found that PEl at $6 \mu \mathrm{g} /$ $\mathrm{ml}$ (total volume $0.5 \mathrm{ml}$ in a 24-well plate) resulted in $81 \%$ relative cell viability (Figure 1A). In addition, we also investigated the effect of PEI/DNA ratios ( $\mathrm{w} / \mathrm{w}$ ) and types of transfection on the transfection efficiency. Prior to the addition to cells, PEI $(3 \mu \mathrm{g})$ was mixed with different masses of pCITE (eGFP) $(\mu \mathrm{g})$ in tubes at prefixed ratios. It was found that the highest percentage 
of cells emitting fluorescence was observed at a PEI/DNA ratio of 1:1.5 in both forward and reverse transfection (Figure 1B). The percentage gradually reduced at a higher $\mathrm{PEI} / \mathrm{DNA}$ ratio. It is interesting to note that the optimal ratio obtained ( $6 \mu \mathrm{g}$ of PEl: $9 \mu \mathrm{g}$ of DNA $/ \mathrm{ml}$ ) was different compared with that of other studies [11,12] using Chinese hamster ovary cells. Higher DNA concentration was used in this study because low DNA concentration led to few or undetectable fluorescing cells (data not shown). The percentage of cells emitting GFP in this study was also relatively low $(<30 \%)$ compared with that in Chinese hamster ovary cells, which can be as high as $70-80 \%$ [11]. This suggests that different cell type plays an important role in this case.

Using the optimized parameters, we proceeded with the transfection of plasmid cocktail into the BSR T7/5 cells to produce the rNDV harboring the GFP gene. Transfection using Lipofectamine 3000 was included for a comparison. The total mass of DNA added for transfection was $4.5 \mu \mathrm{g}$ in a $0.5-\mathrm{ml}$ culture media. Lipofectamine 3000 transfection successfully rescued rNDV for both forward and reverse transfection, whereas PEI was successful only once (with forward transfection) in a 24-well plate (Figure 2).

The experiment was then upscaled from a 24-well plate to a 6-well plate, as commonly described [2]. Therefore, the previously mentioned optimization parameters were scaled up accordingly. The transfections using both transfection reagents were successful as shown in Figure 3, albeit with inconsistent success rates (Table 1). This could be owing to the high plasmid DNA (pDNA) mass $(22.5 \mu \mathrm{g})$ used in the transfection, which could lead to cytotoxicity. To investigate this, we reduced the total pDNA mass to $1.8 \mu \mathrm{g}$, a mass that has been optimized for Lipofectamine 3000 in most laboratories for the rescue of rNDV [2,13]. The success rate of transfection was $100 \%$ in forward transfection and approximately $90 \%$ in reverse transfection (Figure 4 \& Table 1). However, viral rescue was not successful at all when $\mathrm{PEI}$ was used.

The culture media was harvested to detect the presence of viral progenies. Rather than using the standard plaque

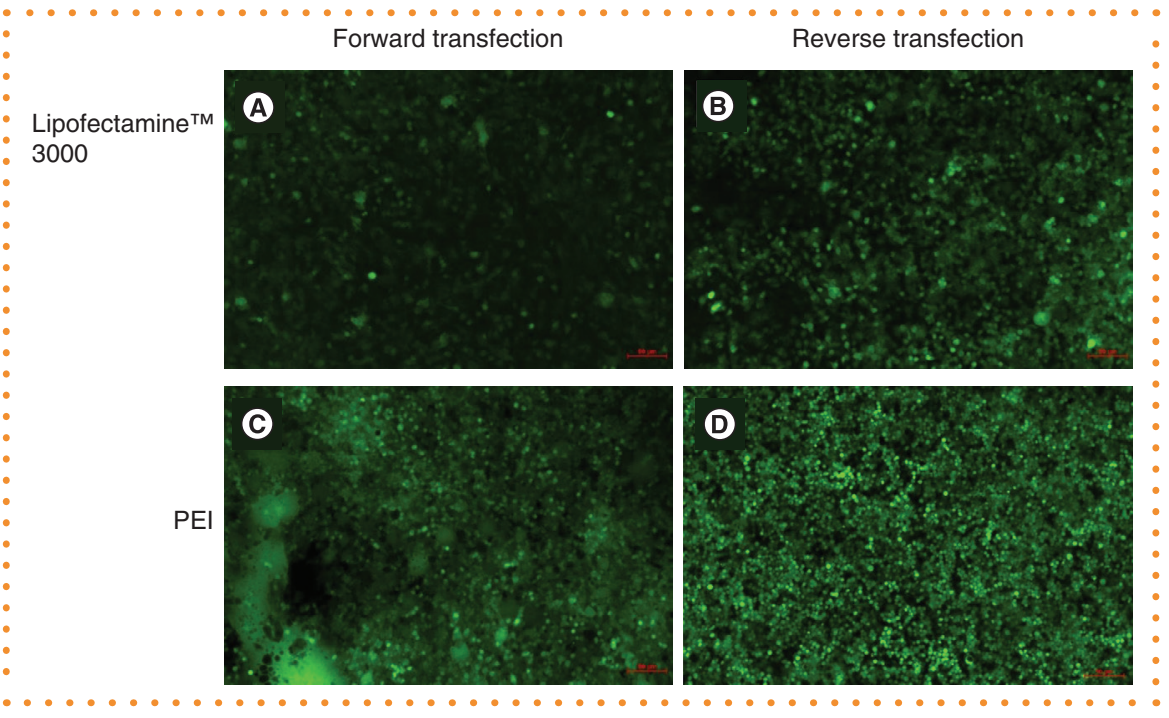

Figure 3. Successful viral rescue using different types of transections and transfection reagents in a 6-well plate. (A) Forward transfection, Lipofectamine ${ }^{\mathrm{TM}}$ 3000. (B) Reverse transfection, Lipofectamine 3000. (C) Forward transfection, PEI. (D) Reverse transfection, PEl. Cell seeding density: $5.05 \times 10^{5}$ cells/well, PEl mass ( $15 \mu \mathrm{g}$ in $2.5 \mathrm{ml}$ of media) and the total plasmid DNA (pDNA) mass was $22.5 \mu \mathrm{g}$ (at a ratio of 1:0.4:0.2:0.2 for pOLTV5(rAF-GFP), pCl-neo(NP), pCl-neo(P), and $\mathrm{pCl}-\mathrm{neo}(\mathrm{L})$ ). $\mathrm{rNDV}$-emitting fluorescence was observed via fluorescence microscope at $100 \times$ magnification. Rescue of rNDV was repeated in three independent biological replicates (each with three independent technical replicates).

PEl: Polyethylenimine; rNDV: Recombinant Newcastle disease virus.

assay, we developed a relatively simple and straight-forward method for the quantification of rNDV, which harbors a fluorescent reporter protein transgene in its genome and expresses the reporter protein during viral

replication. The titer of virus rescued from both forward and reverse transfection using Lipofectamine 3000 was calculated and is shown in Figure 5. Although not statistically significant, there is a trend of higher virus

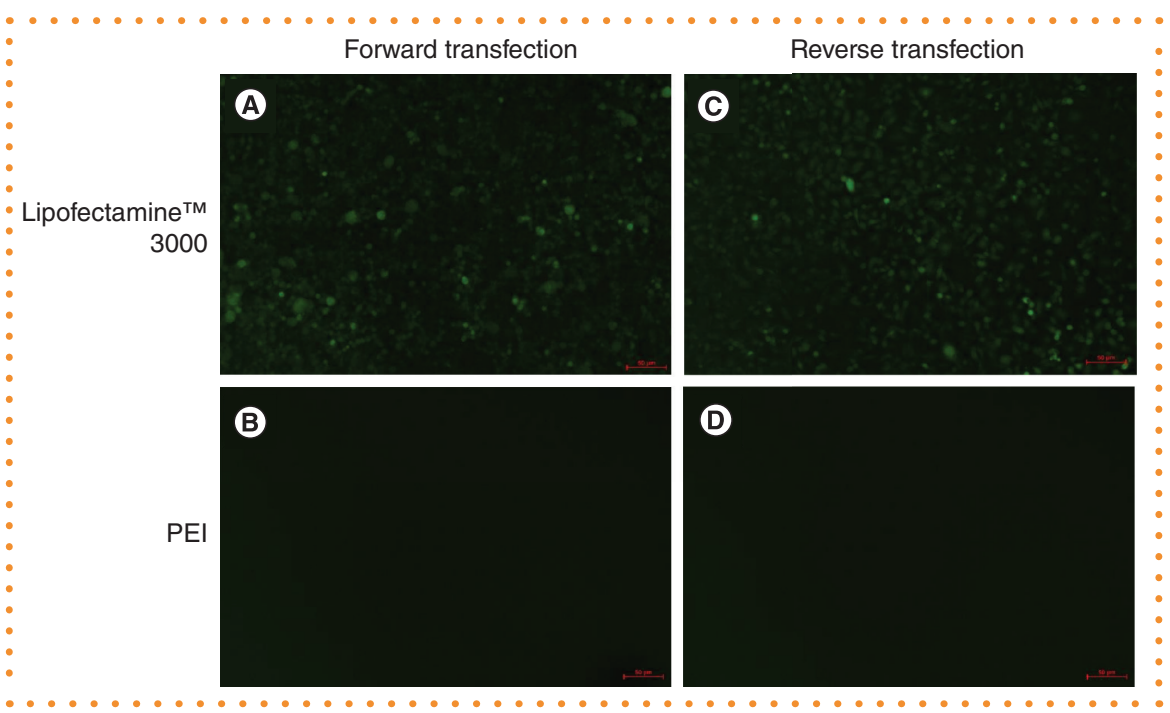

Figure 4. Viral rescue using different types of transfections and transfection reagents with reduced DNA mass of $1.8 \mu \mathrm{g}$ in a 6-well plate. (A) Forward transfection, Lipofectamine ${ }^{T M} 3000$. (B) Reverse transfection, Lipofectamine 3000. (C) Forward transfection, PEI. (D) Reverse transfection, PEI. All wells were observed at 100× magnification under a fluorescence microscope. Rescue of recombinant Newcastle disease virus was repeated in three independent biological replicates (each with three independent technical replicates).

PEI: Polyethylenimine. 


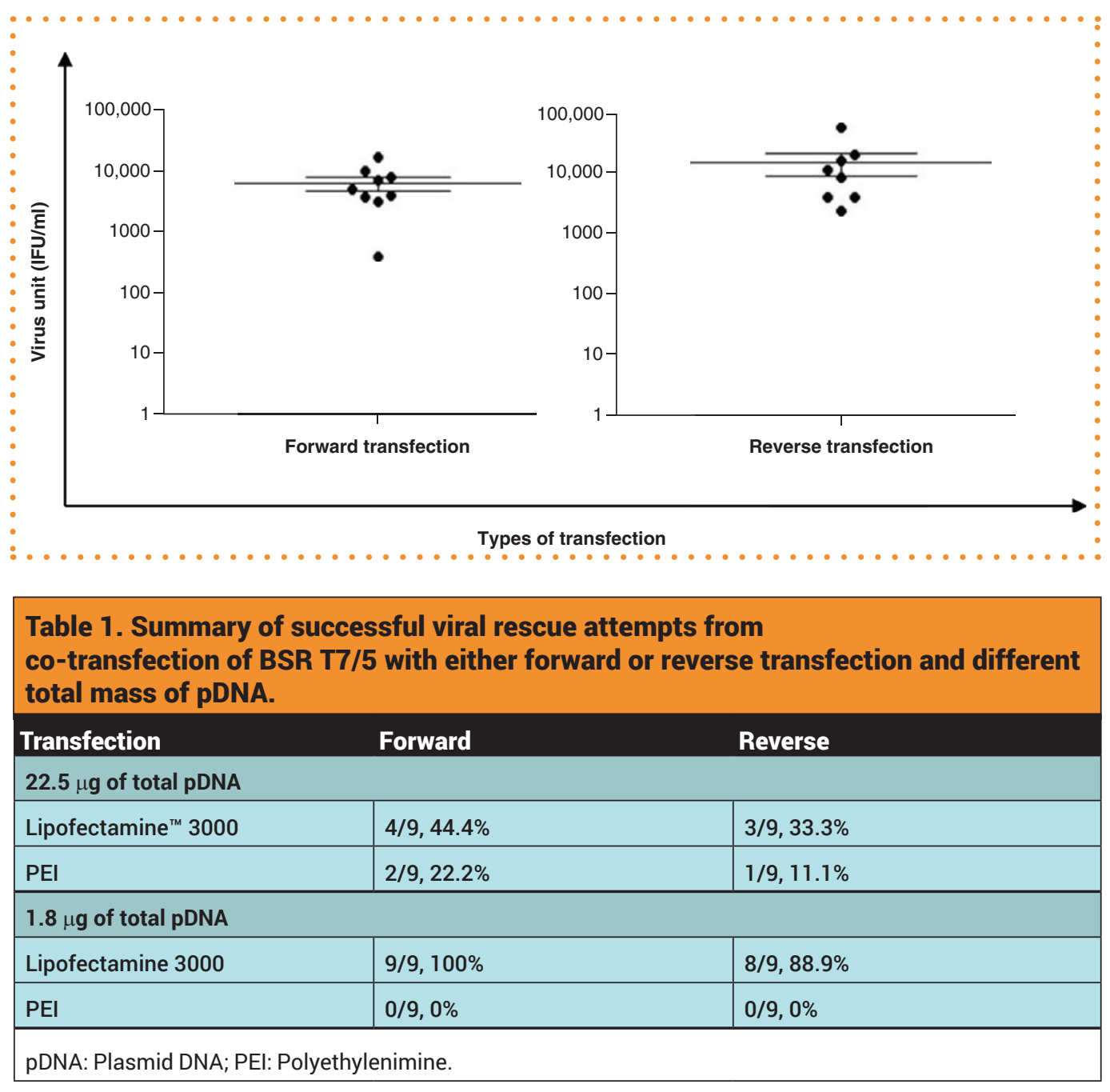

titer using reverse transfection compared with forward transfection.

In conclusion, we have shown that the virus could be rescued in both transfection methods; however, reverse transfection prevailed the standard forward transfection with a twofold increase in virus titer, albeit insignificant statistically. Despite low success rates in transfection, $\mathrm{PEI}$ could be used to rescue NDV via reverse genetics. We have also managed to establish a more rapid and accurate method to quantitate infectious NDV titer compared with standard plaque assays and hemagglutination assays.

\section{FUTURE PERSPECTIVE}

To our knowledge, there are no reports describing the rescue of NDV using PEI as a transfection reagent for plasmids. The success of rescuing NDV in our system showed that PEI could be used as
Figure 5. Virus quantification was performed by infecting HT29 cells with culture media harvested from the well. $\mathrm{n}=9$ for forward transfection, whereas $\mathrm{n}=\mathbf{8}$ for reverse transfection. The number of samples (n) was based on the attempts of previous successful rescues using Lipofectamine $\mathrm{T}^{\mathrm{TM}} 3000$ and $1.8 \mu \mathrm{g}$ of total pDNA. an alternative to Lipofectamine. PEI reduces the cost of production for rescuing NDV in large scale. In addition, the newly established virus titration using flow cytometry provides rapid yet accurate quantification of the infectious GFP-expressing virus.

\section{AUTHOR CONTRIBUTIONS}

P-S Cheow conducted the experiments and prepared the manuscript. TK Tan, AA-L Song, KYusoff and SL Chia designed the study. All authors reviewed and revised the manuscript.

\section{ACKNOWLEDGMENTS}

The authors would like to thank the administrative personnel in the Faculty of Biotechnology and Biomolecular Sciences, Universiti Putra Malaysia, for their contribution with the ordering and purchasing of chemicals and consumables for this research.

\section{FINANCIAL \& COMPETING INTERESTS DISCLOSURE}

This work was supported by the Ministry of Education, Malaysia, research grant (FRGS/1/2017/STG05/UPM/02/6) and Universiti Putra Malaysia, Graduate Research Fellowship for P-S Cheow. The authors have no other relevant affiliations or financial involvement with any organization or entity with a financial interest in or financial conflict with the subject matter or materials discussed in the manuscript apart from those disclosed.

No writing assistance was utilized in the production of this manuscript.

\section{OPEN ACCESS}

This work is licensed under the AttributionNonCommercial-NoDerivatives 4.0 Unported License. To view a copy of this license, visit http://creativecommons.org/licenses/ by-nc-nd/4.0/ 


\section{REFERENCES}

Papers of special note have been highlighted as: • of interest; $\cdot \bullet$ of considerable interest

1. Kapczynski DR, Afonso CL, Miller PJ. Immune responses of poultry to Newcastle disease virus. Dev. Comp. Immunol. 41(3), 447-453 (2013).

2. Ayllon J, García-Sastre A, Martínez-Sobrido L. Rescue of recombinant Newcastle disease virus from cDNA. J. Vis. Exp. Jove. 80, e50830 (2013).

- A video is included in this study that teaches the technique to rescue recombinant Newcastle disease virus by using reverse genetics.

3. Liu H, Albina E, Gil P, Minet C, de Almeida RS. Two-plasmid system to increase the rescue efficiency of paramyxoviruses by reverse genetics: the example of rescuing Newcastle disease virus. Virology 509, 42-51 (2017).

4. Peeters B, de Leeuw O. A single-plasmid reverse genetics system for the rescue of non-segmented negative-strand RNA viruses from cloned full-length cDNA. J. Virol. Methods 248, 187-190 (2017).

- Describes the methods used to rescue viruses via a single-plasmid system by improving the transfection efficiency.

5. Milián E, Julien T, Biaggio R et al. Accelerated mass production of influenza virus seed stocks in HEK-293 suspension cell cultures by reverse genetics. Vaccine 35(26), 3423-3430 (2017).

-. Describes the rescue of influenza virus via reverse genetics and polyethylenimine (PEI) as a transfection reagent.

6. Kalyanasundram J, Hamid A, Yusoff K, Chia SL. Newcastle disease virus strain AF2240 as an oncolytic virus: a review. Acta Trop. 183, 126-133 (2018).

7. Peeters BP, de Leeuw OS, Koch G, Gielkens AL. Rescue of Newcastle disease virus from cloned cDNA: evidence that cleavability of the fusion protein is a major determinant for virulence. J. Virol. 73(6), 5001-5009 (1999).

.- The cell line used for recombinant Newcastle disease virus rescue, BSR T7/5 via reverse genetics is identical to the cell line used in this study.

8. Mossman T. Rapid colorimetric assay for cellular growth and survival: application to proliferation and cytotoxicity assays. J. Immunol. Methods 65(1-2), 55-63 (1983).

9. Okon EU, Hammed G, El Wafa PA, Abraham O, Case N, Henry E. In vitro cytotoxicity of polyethyleneimine on HeLa and Vero cells. Int. J. Innov. Appl. Stud. 5(3), 192 (2014).

-. Results displayed in the study gave an idea on what range the cells can tolerate with the PEl concentration.

10. Yang HJ, Feng P, Wang L et al. Caveolin-1 mediates gene transfer and cytotoxicity of polyethyleneimine in mammalian cell lines. Mol. Cell. Biochem. 402(1-2), 203-211 (2015)

11. Xie Q, Xinyong G, Xianjin C, Yayu W. PEI/DNA formation affects transient gene expression in suspension Chinese hamster ovary cells via a one-step transfection process. Cytotechnology 65(2), 263-271 (2013).

- Investigates the effect of the PEI/DNA ratio on transfection efficiency, which is very similar to our study.

12. Sang $Y, X i e K, M u Y$ et al. Salt ions and related parameters affect $\mathrm{PEI}-\mathrm{DNA}$ particle size and transfection efficiency in Chinese hamster ovary cells. Cytotechnology 67(1), 67-74 (2015).

- Investigates the DNA/PEI ratio, which exhibits the highest transfection efficiency.

13. Santry LA, McAusland TM, Susta L et al. Production and purification of high-titer Newcastle disease virus for use in preclinical mouse models of cancer. Mol. Ther. Methods Clin. Dev. 9, 181-191 (2018).

- A section in the article describes the methodology of rescuing Newcastle disease virus via transfection. 